\title{
Prolonged complete response following gemcitabine-erlotinib combined therapy in advanced pancreatic cancer
}

\author{
ANNA M. CZARNECKA ${ }^{1}$, PIOTR KORZEN' ${ }^{1}$, ANNA NOWAK-DEMENT ${ }^{1}$, \\ WOJCIECH KUKWA $^{2}$, JAN KORNILUK ${ }^{1}$ and CEZARY SZCZYLIK ${ }^{1}$ \\ ${ }^{1}$ Department of Oncology, Military Institute of Medicine, Warsaw 04-141; ${ }^{2}$ Department of Otolaryngology, \\ Czerniakowski Hospital, Medical University of Warsaw, Warsaw 00-739, Poland
}

Received April 6, 2014; Accepted December 19, 2014

DOI: $10.3892 / \mathrm{ol} .2015 .4009$

\begin{abstract}
Pancreatic cancer is one of the most lethal types of malignant solid tumor and is typically associated with a poor prognosis. The majority of patients are diagnosed with advanced-stage disease, therefore, the median survival period is $<6$ months. Recently, a number of basic research projects and clinical trials were undertaken with the aim of improving treatment outcomes in pancreatic cancer; however, only one agent, erlotinib, passed the clinical trials. Erlotinib is an inhibitor of epidermal growth factor receptor, which when overexpressed in cancer, promotes angiogenesis, cell proliferation and inhibits apoptosis. The US Food and Drug Administration and European Medicines Agency approved erlotinib in combination with gemcitabine for the first-line treatment of advanced pancreatic cancer. To the best of our knowledge, the current study is the first to report a case of pancreatic cancer treated with this regimen alone to achieve a complete response (CR). A 40-year-old male with a medical history of chronic pancreatitis and hypertension was diagnosed with medically inoperable adenocarcinoma of the pancreas. Following palliative surgery, the patient began palliative gemcitabine and erlotinib chemotherapy. After three months, this treatment strategy resulted in a CR, as determined by imaging studies. Therapy was discontinued after 14 months due to the development of peritoneal metastases and the patient was referred for treatment with the folinic acid, 5-fluorouracil, irinotecan and oxaliplatin regimen. A CR is rarely reported in pancreatic cancer, however, a treatment strategy of gemcitabine and erlotinib may induce rapid regression of advanced-stage disease.
\end{abstract}

\section{Introduction}

Pancreatic cancer is the fifth leading cause of cancer-related mortality in Europe, with a variable mortality rate of

Correspondence to: Dr Anna M. Czarnecka, Department of Oncology, Military Institute of Medicine, 128 Szaserow Street, Warsaw 04-141, Poland

E-mail: anna.czarnecka@gmail.com

Key words: pancreatic neoplasms, erlotinib, gemcitabine, neoplasm regression, pancreatic cancer
6.6-8.2/100,000 males and 4.0-5.7/100,000 females. Lung cancer incidence and mortality increases in females, while pancreatic cancer mortality rises in the general population. At the same time, for the majority of other cancer types, the mortality rate is decreasing $(1,2)$. Pancreatic cancer is associated with a number of genetic factors, including germline mutations in BRCA2, p16/CDKN2A, PRSS1, STK11/LKB1 and DNA mismatch repair genes, as well as allergies, long-term pancreatitis and cigarette smoking (3). Furthermore, chlorinated hydrocarbons and polycyclic aromatic hydrocarbons are reported to be occupational risk factors (4). A recent meta-analysis determined that multidetector-computed tomography (CT) and magnetic resonance imaging/cholangiopancreatography have comparable sensitivity and specificity rates for the diagnosis and staging of pancreatic cancer. Endoscopic ultrasound exhibits the most favorable sensitivity and specificity rates for lesions measuring $<2 \mathrm{~cm}$, and improved staging has been observed when positron emission tomography (PET)-CT scans are included in the evaluation (5). PET/CT detects distant metastases not documented by CT, thus affecting the treatment strategy. PET/CT is also able to monitor the treatment efficacy, thus identifying metabolic responses to treatment that are not detected by CT (6). Due to poor chemotherapy outcomes, surgical resection remains the primary treatment strategy for pancreatic cancer. Recently, an improved understanding of pancreatic tumor biology allowed the development of the novel chemotherapeutic agent, erlotinib. Erlotinib, an inhibitor of epidermal growth factor receptor (EGFR; ERBB1; HER1), passed clinical trials and with gemcitabine, is now approved for the first-line treatment of advanced-stage pancreatic cancer (7). The current study presents the case of a 40-year-old male who was diagnosed with medically inoperable pancreatic adenocarcinoma and was treated with palliative gemcitabine and erlotinib chemotherapy. The patient provided written informed consent.

\section{Case report}

A 40-year-old male with a medical history of chronic pancreatitis and hypertension was admitted to the Deaprtment of Surgery, Military Institute of Medicine (Warsaw, Poland) in July 2011 due to cholestasis. The patient underwent an endoscopic retrograde cholangiopancreatography, with the 
insertion of stents into the pancreatic and common bile ducts. With the exception of a marginally dilated Wirsung's duct (diameter, $5 \mathrm{~mm}$ ), CT of the abdomen revealed no abnormalities; however, an endoscopic ultrasound procedure performed in August 2011 identified a poorly-defined hypoechogenic mass with irregular borders in the head of the pancreas. The patient underwent palliative open surgery, including a cholecystectomy, a Roux-en-Y hepaticojejunostomy bypass and Braun's enteroanastomosis due to cancer infiltration of the superior mesenteric vein. A subsequent histological analysis of the lesion, which revealed a ductal adenocarcinoma: the tumor formed a firm, poorly defined yellowish mass. In the tumor glandular structures imitating pancreatic ducts were embedded in the abundant desmoplastic stroma. The tumor demonstrated a mixture of medium-sized duct-like and tubular structures of variable shape and incompletely formed glands. Cells with an eosinophilic cytoplasm with a variation in nuclear size, chromatin structure and nucleoli were observed and multiple mitotic figures were reported. Furthermore, a CT scan performed in September 2011 identified ascites, a 26-mm lesion in the pancreas, enlarged duodenal lymph nodes measuring $\leq 12 \mathrm{~mm}$, and splenic and superior mesenteric vein thrombosis (Fig. 1). Therefore, the patient was transferred to the Department of Oncology, Military Institute of Medicine to commence 26 courses of palliative combined chemotherapy with $1,000 \mathrm{mg} / \mathrm{m}^{2}$ intravenous gemcitabine every week and $100 \mathrm{mg}$ erlotinib every day. According to the Common Toxicity Criteria (CTC) (8), grade II neutropenia and grade III leukopenia occurred during treatment, causing courses 4,8 , 13 and 23 to be delayed; these adverse hematological effects were treated with pegfilgrastim at a dose of $6 \mathrm{mg}$ (one dose per cycle). Furthermore, the patient developed a grade II skin rash, according to the CTC. After three months of treatment, in December 2011, a CT scan demonstrated reduced ascites with the absence of any measurable lesions (Fig. 2). In addition, the patient underwent laparotomy one month after CT, which revealed no visible malignant processes, and a PET-CT performed in April 2012 confirmed this favorable treatment outcome (Fig. 3). However, towards the end of April 2012, the patient experienced severe hematemesis caused by esophageal and gastric fundal varices, which had arisen as a result of portal hypertension from a vein thrombosis that had developed as a complication of gemcitabine treatment. Therefore, gemcitabine infusions were rescheduled and now administered every second week. A follow-up CT scan performed in August 2012 demonstrated maintained ascites due to portal hypertension as a side-effect of the therapy. The patient maintained a CR for $>12$ months, however, cancer cells were present in the ascitic fluid in November 2012 and new lesions were identified in a CT scan performed in August 2012. The patient demonstrated disease progression manifested as novel lesions in the peritoneal cavity and was transferred to a folinic acid, 5-fluorouracil (5-FU), irinotecan and oxaliplatin (FOLFIRINOX) chemotherapy regimen in January 2013. The patient progressed rapidly and died in July 2013.

\section{Discussion}

Pancreatic cancer remains one of the most lethal types of tumor and is typically associated with a poor prognosis. The

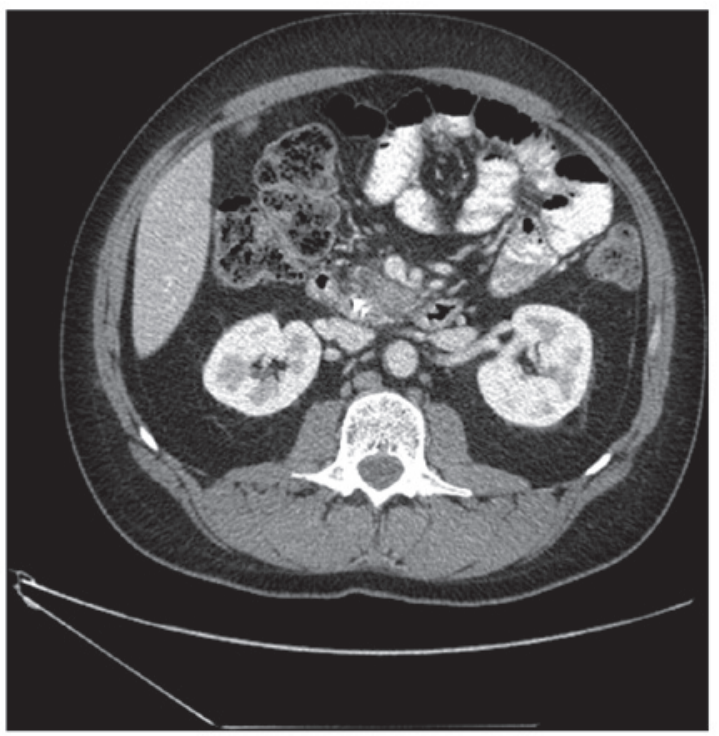

Figure 1. Contrast-enhanced computed tomography scan performed prior to treatment, indicating a lesion in the head of the pancreas.

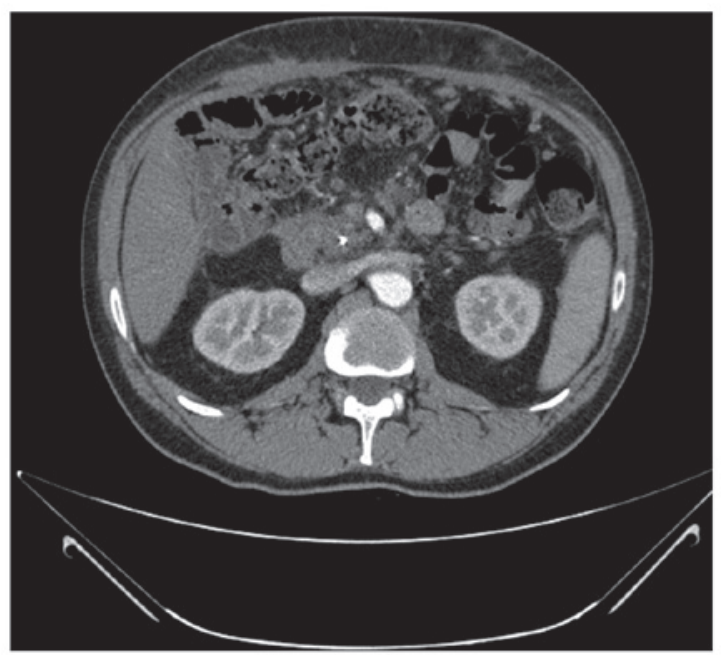

Figure 2. Follow-up contrast-enhanced computed tomography scan performed after chemotherapy indicating that the head of the pancreas is free of the cancerous mass.

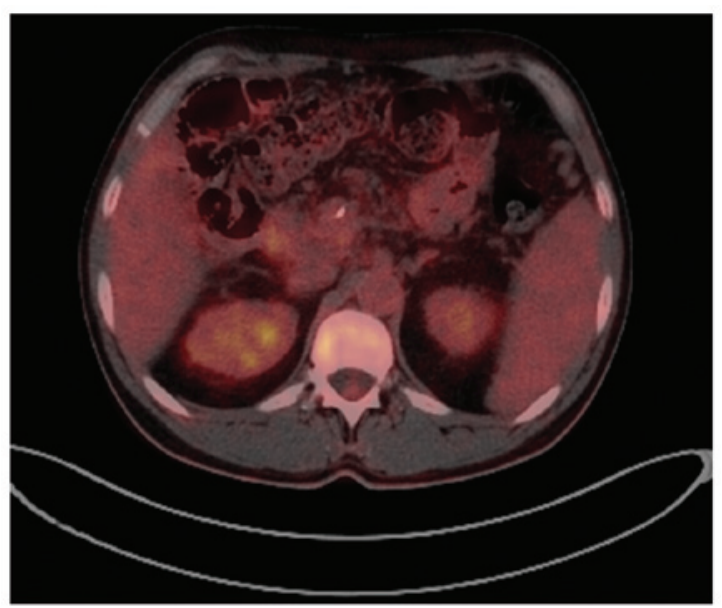

Figure 3. Positron emission tomography-computed tomography scan demonstrating the abdomen without a pathological concentration of radioisotope. 
majority of pancreatic cancer patients are diagnosed with advanced-stage disease, therefore, the median survival period is <6 months. Until 1997, the management of pancreatic cancer was based on a 5-FU regimen; in 1997, Burris et al reported that gemcitabine-treated patients demonstrated significant clinical benefits compared with 5-FU-treated patients, including higher response rates (23.8 vs. $4.8 \% ; \mathrm{P}=0.0022)$, longer median survival times (5.65 vs. 4.41 months; $\mathrm{P}=0.0025)$ and higher one-year survival rates ( 18 vs. $2 \% ; \mathrm{P}=0.0025)$ (9). Trials based on combination therapy with oxiplatin, 5-FU, irinotecan, pemetrexed, capacetabine and biological agents, such as the farnesyl transferase inhibitor, tipifarnib, and the matrix metalloproteinases, revealed inferior outcomes compared with gemcitabine treatment alone (10). Furthermore, combined therapeutic strategies have exhibited superior outcomes compared with single agent-based molecular-targeted therapies (for example, those targeted to $K R A S, V E G F, V E G F-C$, $N F-\kappa B, H E R-2$ and HER-3 mutations) (11-14). In 2005, a novel chemotherapeutic agent regimen involving erlotinib in combination with gemcitabine was approved for the first-line treatment of advanced-stage pancreatic cancer. A placebo-controlled phase III trial demonstrated that administration of the EGFR inhibitor in combination with gemcitabine is particularly efficient in preventing pancreatic ductal adenocarcinoma patients from developing skin toxicity. However, the EGFR expression levels of tumor cells were not predictive of response in this phase III trial and markers to characterize an erlotinib-responding subgroup are currently unavailable $(15,16)$. Furthermore, a hypothetical managed care plan determined that the addition of erlotinib to the gemcitabine regimen resulted in a low budget impact, estimated at \$0.02 per member per month (17).

Excluding the present study, thus far only two patients receiving 24 cycles of gemcitabine combined with erlotinib for the first-line treatment of pancreatic cancer exhibited a transient CR (18). However, one patient also achieved a CR in a trial using a regimen that consisted of 28-day cycles of gemcitabine treatment $\left(1,200 \mathrm{mg} / \mathrm{m}^{2}\right.$ in 120 -min infusions on days 1, 8 and 15) plus erlotinib (100 mg, orally once daily) (19). In addition, erlotinib single-agent therapy appeared to be an effective treatment strategy for gemcitabine-refractory advanced pancreatic cancer patients, exhibiting a benefit rate of $22 \%$ (20). Recently, the FOLFIRINOX regimen, based on an infusion of 5-FU/folic acid plus irinotecan and oxaliplatin, was reported to be superior to gemcitabine, but only in patients with a good performance status of $90-100 \%$, according to the Karnofsky scale. Compared with gemcitabine treatment alone, the FOLFIRINOX regimen demonstrated improved objective response rates (31.6 vs. 9.4\%; $\mathrm{P}<0.001$ ), progression-free survival [PFS; 6.4 vs. 3.3 months; hazard ratio (HR), 0.47; $\mathrm{P}<0.001$ ], overall survival (OS; 11.1 vs. 6.8 months; HR, 0.57; $\mathrm{P}<0.001$ ) and one-year survival (48.4 vs. 20.6\%); however, a higher toxicity rate with grade III-IV neutropenia (45.7 vs. $21 \%$; $\mathrm{P}<0.001$ ), febrile neutropenia (5.4 vs. $1.2 \% ; \mathrm{P}=0.03$ ), grade III-IV thrombocytopenia, diarrhea and sensory neuropathy were also observed during treatment (21). Although a partial response during gemcitabine-erlotinib combination therapy was observed, cases with a CR were rarely reported. According to Carbonell et al (22), studies conducted over the last decade describe 29 patients who achieved a CR during treatment with only one of the agents (gemcitabine or erlotinib) following chemotherapy alone. In 2014, a phase II study was published to evaluate the combination of treatment with daily erlotinib (100 mg, orally) and weekly gemcitabine $\left(1,000 \mathrm{mg} / \mathrm{m}^{2}\right.$, infused at a rate of $10 \mathrm{mg} / \mathrm{m}^{2} / \mathrm{min}$ ) in a cohort of 46 previously untreated patients with locally advanced, inoperable or metastatic pancreatic cancer (23). The median PFS time was 14 weeks, the one-year OS rate was $20.2 \%$ and the median OS time was 26 weeks, with only five patients (10.9\%) achieving an objective response. Furthermore, the overall disease control rate was $56.5 \%$ and no patients achieved a CR (23). To the best of our knowledge, the current study presents the first reported case of an extended $\mathrm{CR}$ in pancreatic cancer achieved using combination therapy of low-dose gemcitabine $\left(1,000 \mathrm{mg} / \mathrm{m}^{2}\right)$ and erlotinib.

EGFR belongs to the ErbB family of tyrosine kinase receptors and contains four members: ERBB1 (EGFR or HER1), ERBB2 (HER2/neu), ERBB3 (HER3) and ERBB4 (HER4). In pancreatic cancer, ErbB overexpression occurs at a frequency of $30-60 \%$, promoting angiogenesis and cell proliferation, and inhibiting apoptosis $(11,14)$. The EGFR kinase inhibitors, erlotinib and gefitinib, have demonstrated clinical efficacy against pancreatic and non-small cell lung cancer (NSCLC), however, EGFR status in the two diseases does not appear to be associated with disease response or stability (10). In NSCLC, mutations in exons 19 and 21 of the EGFR gene have been associated with improved outcomes (24); however, the presence of the same mutations (exons 19 and 21) in pancreatic cancer is rare, with an incidence of $2 \%$, and has demonstrated no effect on erlotinib activity in the clinical setting (25). Furthermore, the presence of skin toxicity, improved performance status and lower pain intensity scores have been associated with greater OS in patients with pancreatic cancer, whereas age and comorbidity have not $(15,26)$. Additional studies are required to understand the underlying mechanism that leads to skin rashes benefitting OS. In addition, patients with elevated cancer antigen (CA)19-9 levels prior to surgical resection exhibited worse outcomes than patients with CA19-9 levels within the normal range, and CA19-9 levels decreased or normalized by $\geq 20-50 \%$ of the baseline pretreatment levels were associated with a survival benefit (27). Another factor involved in pancreatic treatment outcome is KRAS mutation status, which was determined not to be predictive of the objective response to anti-EGFR treatment with erlotinib. In a post-hoc analysis study of AIO-PK0104, a phase III trial comparing gemcitabine/erlotinib followed by capecitabine with capecitabine/erlotinib followed by gemcitabine for the treatment of advanced pancreatic cancer, KRAS mutation status was verified and no association was identified with objective response $(\mathrm{P}=0.40$; however, wild-type $K R A S$ patients did exhibit improved OS (HR, 1.68; P=0.005) (28). AIO-PK0104 patients were also evaluated for $K R A S$ exon 2 mutations, EGFR expression, phosphatase and tensin homolog (PTEN) expression, and R497 K polymorphisms (PMs) of EGFR intron 1 and exon 13. It was reported that wild-type $K R A S$ status is associated with improved OS (HR, 1.68; $\mathrm{P}=0.005)$, however, no significant OS correlation was identified for EGFR (HR, 0.96) or PTEN (HR, 0.77) overexpression, EGFR amplification (HR, 1.22), or EGFR intron 1 (HR, 0.91) or exon 13 (HR, 0.83) 
R497K PMs. In addition, the expression of none of the six biomarkers investigated correlated with the occurrence of a skin rash (29). Furthermore, a trial conducted by the National Cancer Institute of Canada Clinical Trials Group PA.3 clarified that no correlation exists between PFS-OS data and KRAS mutation status (16).

In conclusion, the present case report indicated that a combination of erlotinib and gemcitabine may be an effective treatment strategy for patients with local advanced pancreatic cancer. However, additional studies are required to identify clinically important molecular markers that may facilitate the prediction of the treatment response, as well as improve our understanding of the biology of this disease, with incorporation of this knowledge into clinical trials. Furthermore, future studies should attempt to identify predictive and prognostic markers to aid in the development and application of therapeutic agents. Finally, control of pancreatic cancer will require a combination of targeted agents and individualized therapies based on tumor genetics, therefore, research such as the recent investigation $(30,31)$ into the mechanisms of drug resistance and the combined targeted agents required in the clinic must continue.

\section{Acknowledgements}

The present study was supported by statutory funding from the Military Institute of Medicine (Warsaw, Poland; grant no. WIM/1/1744).

\section{References}

1. Ferlay J, Parkin DM and Steliarova-Foucher E: Estimates of cancer incidence and mortality in Europe in 2008. Eur J Cancer 46: 765-781, 2010.

2. Malvezzi M, Bertuccio P, Levi F, La Vecchia C and Negri E: European cancer mortality predictions for the year 2012. Ann Oncol 23: 1044-1052, 2012

3. Koorstra JB, Hustinx SR, Offerhaus GJ and Maitra A: Pancreatic carcinogenesis. Pancreatology 8: 110-125, 2008.

4. Andreotti G and Silverman DT: Occupational risk factors and pancreatic cancer: a review of recent findings. Mol Carcinog 51: 98-108, 2012.

5. Shrikhande SV, Barreto SG, Goel M and Arya S: Multimodality imaging of pancreatic ductal adenocarcinoma: a review of the literature. HPB (Oxford) 14: 658-668, 2012.

6. Picchio M, Giovannini E, Passoni P, et al: Role of PET/CT in the clinical management of locally advanced pancreatic cancer. Tumori 98: 643-651, 2012.

7. Moore MJ, Goldstein D, Hamm J, et al; National Cancer Institute of Canada Clinical Trials Group: Erlotinib plus gemcitabine compared with gemcitabine alone in patients with advanced pancreatic cancer: a phase III trial of the National Cancer Institute of Canada Clinical Trials Group. J Clin Oncol 25: 1960-1966, 2007.

8. National Institutes of Health: Common Terminology Criteria for Adverse Events (CTCAE) Version 4.0. May 28, 2009. http:// evs.nci.nih.gov/ftp1/CTCAE/CTCAE_4.03_2010-06-14_ QuickReference_5x7.pdf

9. Burris HAIII,Moore MJ, Andersen J, Green MR, Rothenberg ML, Modiano MR, Cripps MC, Portenoy RK, Storniolo AM, Tarassoff P, Nelson R, Dorr FA, Stephens CD and Von Hoff DD: Improvements in survival and clinical benefit with gemcitabine as first-line therapy for patients with advanced pancreas cancer: a randomized trial. J Clin Oncol 15: 2403-2413, 1997.

10. Burris HA III: Recent updates on the role of chemotherapy in pancreatic cancer. Semin Oncol 32 (4 Suppl 6): S1-S3, 2005.

11. Borja-Cacho D, Jensen EH, Saluja AK, Buchsbaum DJ and Vickers SM: Molecular targeted therapies for pancreatic cancer. Am J Surg 196: 430-441, 2008.
12. Kleespies A, Jauch KW and Bruns CJ: Tyrosine kinase inhibitors and gemcitabine: new treatment options in pancreatic cancer? Drug Resist Updat 9: 1-18, 2006.

13. Longo R, Cacciamani F, Naso G and Gasparini G: Pancreatic cancer: from molecular signature to target therapy. Crit Rev Oncol Hematol 68: 197-211, 2008.

14. Starling N, Neoptolemos J and Cunningham D: Role of erlotinib in the management of pancreatic cancer. Ther Clin Risk Manag 2: 435-445, 2006.

15. Vickers MM, Powell ED, Asmis TR, et al: Comorbidity, age and overall survival in patients with advanced pancreatic cancer - results from NCIC CTG PA.3: a phase III trial of gemcitabine plus erlotinib or placebo. Eur J Cancer 48: 1434-1442, 2012.

16. da Cunha Santos G, Dhani N, Tu D, et al: Molecular predictors of outcome in a phase 3 study of gemcitabine and erlotinib therapy in patients with advanced pancreatic cancer: National Cancer Institute of Canada Clinical Trials Group Study PA.3. Cancer 116: 5599-5607, 2010

17. Danese MD, Reyes C, Northridge K, Lubeck D, Lin CY and O'Connor P: Budget impact model of adding erlotinib to a regimen of gemcitabine for the treatment of locally advanced nonresectable or metastatic pancreatic cancer. Clin Ther 30: 775-784, 2008.

18. Klapdor R, Klapdor S and Bahlo M: Combination therapy with gemcitabine (GEM) and erlotinib (E) in exocrine pancreatic cancer under special reference to RASH and the tumour marker CA19-9. Anticancer Res 32: 2191-2197, 2012.

19. Feliu J, Borrega P, León A, et al: Phase II study of a fixed dose-rate infusion of gemcitabine associated with erlotinib in advanced pancreatic cancer. Cancer Chemother Pharmacol 67: 215-221, 2011.

20. Iyer NIK RV, Tan W, Litwin A, Starostik P, Levea C, Tucker C, Ma W, Fakih M and Adjei AA: A phase II study of erlotinib in patients (PTS) with advanced pancreatic cancer (APC) who are refractory to gemcitabine $(\mathrm{G})$. ASCO Gastrointestinal Cancers Symposium: abstract 258, 2010.

21. Heinemann V, Haas M and Boeck S: Systemic treatment of advanced pancreatic cancer. Cancer Treat Rev 38: 843-853, 2012.

22. Carbonell S, Espinosa J, Zarco A, et al: Complete pathological response after chemotherapy alone in a patient with pancreatic adenocarcinoma. Pancreas 41: 657-659, 2012.

23. Renouf DJ, Tang PA, Hedley D, Chen E, Kamel-Reid S, Tsao MS, Tran-Thanh D, Gill S, Dhani N, Au HJ, Wang L and Moore MJ: A phase II study of erlotinib in gemcitabine refractory advanced pancreatic cancer. Eur J Cancer 50: 1909-1915, 2014.

24. Abdel-Wahed MM, Asaad NY and Aleskandarany M: Expression of matrix metalloproteinase-2 in renal cell carcinoma. J Egypt Natl Canc Inst 16: 168-177, 2004.

25. Riely GJ, Politi KA, Miller VA and Pao W: Update on epidermal growth factor receptor mutations in non-small cell lung cancer. Clin Cancer Res 12: 7232-7241, 2006.

26. Weiss GA, Rossi MR, Khushalani NI, et al: Evaluation of phosphatidylinositol-3-kinase catalytic subunit (PIK3CA) and epidermal growth factor receptor (EGFR) gene mutations in pancreaticobiliary adenocarcinoma. J Gastrointest Onco 4: 20-29, 2013.

27. Wacker B, Nagrani T, Weinberg J, Witt K, Clark G and Cagnoni PJ: Correlation between development of rash and efficacy in patients treated with the epidermal growth factor receptor tyrosine kinase inhibitor erlotinib in two large phase III studies. Clin Cancer Res 13: 3913-3921, 2007.

28. Ballehaninna UK and Chamberlain RS: The clinical utility of serum CA 19-9 in the diagnosis, prognosis and management of pancreatic adenocarcinoma: An evidence based appraisal. J Gastrointest Oncol 3: 105-119, 2012.

29. Boeck S, Jung A, Laubender RP, et al: KRAS mutation status is not predictive for objective response to anti-EGFR treatment with erlotinib in patients with advanced pancreatic cancer. J Gastroenterol 48: 544-548, 2013.

30. Boeck S, Jung A, Laubender RP, et al: EGFR pathway biomarkers in erlotinib-treated patients with advanced pancreatic cancer: translational results from the randomised, crossover phase 3 trial AIO-PK0104. Br J Cancer 108: 469-476, 2013.

31. Renouf D and Moore M: Evolution of systemic therapy for advanced pancreatic cancer. Expert Rev Anticancer Ther 10: 529-540, 2010. 